\title{
Role of transurethral resection of the prostate in the management of prostate cancer
}

\author{
Szollosi Attila, Martha Orsolya, Denes Lorand, Vida Arpad Oliver, Maier Adrian, Pavai Zoltan \\ University of Medicine and Pharmacy Tirgu Mures, Romania
}

\begin{abstract}
Introduction: Prostate cancer is the second most diagnosed cancer in men, after lung cancer. The gold standard procedure in prostate cancer $(\mathrm{PCa})$ diagnosis is the ultrasound guided prostate biopsy. Transurethral resection of the prostate (TURP) used in solving the bladder outlet obstruction, can have a role in detection of PCa. The aim of this retrospective study is to examine the role of transurethral resection of the prostate in the diagnosis and therapy of prostate cancer.

Materials and methods: At the Urology Clinic of Targu Mures we performed a total of 474 TURP over a two year period (2011-2013). The patients had a mean age of 71.857 years, and the indications were PCa with bladder outlet obstruction or bladder outlet obstruction with unknown causes but some with suspicion of PCa.

Results: In case of 474 patients with TURP performed for bladder outlet obstruction, the histopathology findings were the following: 61 cases with newly diagnosed PCa, some in spite of normal PSA values, 23 cases with already hormone treated prostate cancer, while in 50 cases TURP was preceded by prostate biopsy (in 8 cases with increased PSA and several negative biopsies, we could confirm PCa of the peripheral zone of the prostate).

Conclusion: TURP remains the elective surgical therapy of the bladder outlet obstruction, caused by BPH and even prostate cancer. Obtaining a greater volume of prostate tissue can help in the detection of prostate cancer in its early stages, especially in the transitional zone.
\end{abstract}

Keywords: prostate cancer, transurethral resection of the prostate, prostate specific antigen, bladder outlet obstruction, transrectal ultrasound guided prostate biopsy

Received: 20 October 2015 / Accepted: 05 January 2016

\section{Introduction}

Prostate cancer is the most frequently diagnosed cancer in men after bronchopulmonary cancer. The incidence of prostate cancer has increased with the use of prostate specific antigen (PSA) and the digital rectal examination as screening procedures for prostate cancer $[1,2]$.

The gold standard procedure in prostate cancer diagnosis is the ultrasound guided prostate biopsy, performed in case of clinical and laboratory suspicion. Based on literature, detection rate of prostate cancer with sextant ultrasound guided prostate biopsy is $25-30 \%[1,2]$.

Transurethral resection of the prostate (TURP) is the main treatment modality used in surgery of the bladder outlet obstruction, caused by prostate pathology like benign prostatic hyperplasia $(\mathrm{BPH})$ and prostate cancer (PCa) $[3,4]$. It also has a diagnostic role, being an auxiliary method used in conjunction with prostate biopsy, for confirmation of prostate cancer in case of laboratory suspicion (increased PSA levels and/or positive velocity), but with previously negative prostate biopsies $[4,5,6]$. It may be the primary intervention in case of accidental detection of prostate cancer in the resected prostate tissue, in cases with no preoperative clinical or laboratory suspicion of prostate cancer. Based on Ornstein writings, detection rate of prostate cancer with TURP performed in patients with bladder outlet obstruction is $15 \%[3,4]$.

* Correspondence to: Attila Szollosi
The aim of this paper is to examine the role and place of transurethral resection of the prostate in the diagnosis of prostate cancer and therapy of bladder outlet obstruction caused by prostate cancer at the Urology Clinic of Targu Mures.

\section{Materials and Methods}

At the Urology Clinic of Targu Mures we performed a total of 474 TURP surgeries between 1 January 2011 and 1 April 2013, for bladder outlet obstruction, caused by prostate pathology. This retrospective study presents cases of patients, who underwent TURP surgery, over this two year period (2011-2013), for therapeutic and diagnostic reasons, whose histology findings confirmed prostate cancer in the resected prostate tissue, some were with clinical and laboratory suspicion of prostate cancer, but with previews negative prostate biopsies.

We divided this cases into group A [401 patients (84.599\%) - TURP was performed for therapeutically reason for bladder outlet obstruction caused by BPH (with normal PSA level)], group B [23 patients (4.852\%) TURP was performed for therapeutically reason for bladder outlet obstruction caused by prostate cancer under oncological treatment] and group C [50 patients (10.548\%) - TURP with therapeutically and diagnostically reason for bladder outlet obstruction with unknown cause (with elevated PSA levels, but with several negative prostate biopsies)] (Table I).

TURP surgery is an endoscopic procedure, performed under spinal anesthesia during which the inside of prostate 
Table I: Clinical diagnosis

\begin{tabular}{lccccc}
\hline & Clinical diagnosis & 2011 & 2012 & 2013 & Total \\
\hline Group A & BPH + BOO & 138 & 225 & 38 & 401 \\
Group B & Prostate cancer + BOO & 6 & 15 & 2 & 23 \\
Group C & $\begin{array}{c}\text { BOO with } \\
\text { unknown causes } \\
(\text { PSA } \uparrow, \text { BPH?, CaP?) }\end{array}$ & 26 & 20 & 4 & 50 \\
\hline
\end{tabular}

tissue is resected to the prostatic capsule, resulting in numerous prostate fragments. The number of fragments depends on the resected prostate volume. Resected fragments are preserved in $10 \%$ formalin and sent to the Department of Pathology for histopathological examination.

At our clinic, the transrectal prostate biopsy is performed with ultrasound guidance under local anesthesia with lidocaine $10 \%$ spray or spinal anesthesia depending on co-morbidities. In terms of the number of punctures, biopsies performed on the patient, we perform between 8-6-12 biopsies, or in some cases we made saturated biopsies up to 14-16, depending on the volume of the prostate and the patient's age $[7,8]$.

\section{Results}

A total of 474 TURP surgeries were performed at the Urology Clinic of Targu Mures during this two year period (2011-2013). Patients enrolled in this study were aged between 50 and 91 years, with a mean age of 71.857 years (Table II).

The studied patients presented with voiding (obstructive) lower urinary complaints, chronic incomplete / complete urine retention, some with signs of a secondary bladder stones, and some with hematuria. The therapeutic option aimed at solving the bladder outlet obstruction was TURP surgery for all of the cases, preceded by a prostate biopsy in 50 cases (group C). In four cases we also performed transurethral resection of the bladder (TURB) for the suspicion of associated bladder tumors (Table III).

Table II: Age of patients

\begin{tabular}{lc}
\hline Age groups & Number of patients \\
\hline $50-55$ & 27 \\
$56-60$ & 36 \\
$61-65$ & 130 \\
$66-70$ & 95 \\
$71-75$ & 95 \\
$76-80$ & 63 \\
$81-85$ & 5 \\
$86-90$ & 5 \\
$91-95$ & \\
\hline
\end{tabular}

Table III: Surgical interventions

\begin{tabular}{lcccc}
\hline Surgical interventions & 2011 & 2012 & 2013 & Total \\
\hline TURP & 126 & 155 & 17 & 298 \\
„Release” TURP & 6 & 15 & 2 & 23 \\
TURP + Prostate biopsy & 80 & 46 & 23 & 149 \\
TURP + TURB & 1 & 1 & 0 & 2 \\
TURP + TURB + Prostate biopsy & 1 & 1 & 0 & 2 \\
\hline
\end{tabular}

The number of prostate fragments resected during TURP surgery ranged from 4 to 115 fragments. Resection time was up to one hour to prevent TUR syndrome.

In terms of histological findings, in 78 cases of the examined cases (16.455\%) histopathology confirmed prostate cancer in the resected tissue. All of the cases were adenocarcinomas.

In majority of cases, histopathology infirmed prostate cancer in 396 out of 474 cases (83.544\%) [389 cases $(82.067 \%)$ with benign prostatic hyperplasia and normal prostatic tissue, some cases with chronic prostatitis, and 4 cases $(0.843 \%)$ of urothelial carcinoma]. There were 2 cases $(0.421 \%)$ with precancerous states of the prostate [one case with ASAP (Atypical Small Acinar Proliferation) and one case with LGPIN (Low grade prostatic intraepithelial neoplasia)] and one case $(0.211 \%)$ of poorly differentiated carcinoma with neuroendocrine differentiation. Histopathological results are listed in Table IV.

In terms of Gleason score, our 78 cases with prostate cancer had a Gleason score between 4 and 10; in 9 cases the differentiation of the cancer was unclassified (Table V).

In five cases from group A (total of 401), the histological result confirmed a small outbreak of prostate adenocarcinoma of the transition zone with Gleason scores of 2 to 6 .

In four patients, known with prostate cancer from group B (23 patients) who underwent therapeutic TURP, the histological findings showed no prostate cancer in the resected tissue (the result was benign prostatic hyperplasia in three cases and normal prostatic tissue in one case).

Table IV: Histopathology results

\begin{tabular}{lcccc}
\hline Histopathology results & Group A & Group B & Group C & Total \\
\hline $\begin{array}{l}\text { Small focus of prostate adenocar- } \\
\text { cinoma }\end{array}$ & 13 & 1 & 1 & 15 \\
$\begin{array}{l}\text { Prostate adenocarcinoma } \\
\text { BPH + Normal prostatic tissue + }\end{array}$ & 18 & 16 & 29 & 63 \\
Chronic prostatitis & 369 & 4 & 16 & 389 \\
ASAP & 0 & 0 & 1 & 1 \\
LGPIN & 1 & 0 & 0 & 1 \\
$\begin{array}{l}\text { Urothelial carcinoma } \\
\begin{array}{l}\text { Poorly differentiated carcinoma } \\
\text { with neuroendocrine differentiation }\end{array}\end{array}$ & 0 & 0 & 1 & 1 \\
\end{tabular}


In eight cases from group C (50 patients) despite the negative biopsies, which preceded TURP, prostate cancer of the peripheral zone (Gleason scores 7 to 9) was detected in the resected tissue (Table VI).

Table V: Distribution of prostate cancer cases according to Gleason score

\begin{tabular}{lcccc}
\hline Gleason Score & 2011 & 2012 & 2013 & Total \\
\hline $2+2=4$ & 1 & 2 & 0 & 3 \\
$2+3=5$ & 0 & 1 & 0 & 1 \\
$3+3=6$ & 2 & 4 & 2 & 8 \\
$3+4=7$ & 2 & 6 & 1 & 9 \\
$4+3=7$ & 4 & 4 & 0 & 8 \\
$4+4=8$ & 8 & 10 & 3 & 21 \\
$4+5=9$ & 4 & 5 & 0 & 9 \\
$5+4=9$ & 6 & 2 & 0 & 8 \\
$5+5=10$ & 2 & 0 & 0 & 2 \\
Unclassified & 0 & 8 & 1 & 9 \\
& 29 & 42 & 7 & 78 \\
\hline
\end{tabular}

Table VI: Histopathology types and localization of the prostate adenocarcinoma

\begin{tabular}{lc}
\hline $\begin{array}{l}\text { Histopathology types and localization of prostate adenocar- } \\
\text { cinoma }\end{array}$ & $\begin{array}{c}\text { Nr. of } \\
\text { cases }\end{array}$ \\
\hline $\begin{array}{l}\text { Adenocarcinoma of the transitional zone of the prostate } \\
\text { Adenocarcinoma of the peripheral zone of the prostate }\end{array}$ & 3 \\
$\begin{array}{l}\text { Adenocarcinoma of the transition and the peripheral zone of } \\
\text { the prostate }\end{array}$ & 5 \\
Ductal adenocarcinoma & 6 \\
Acinar adenocarcinoma & 40 \\
Ductal with acinar adenocarcinoma & 1 \\
Adenocarcinoma with perineural invasion & 16 \\
Adenocarcinoma with „signet ring cell” & 1 \\
\hline
\end{tabular}

\section{Discussion}

In the daily urological practice, we frequently meet patients with increased PSA levels and negative prostate biopsies [2]. In spite of re-biopsies, the histopathological findings still remain negative, and the patient presents bladder outlet obstruction. What to do? Based on our clinical practice we realize that there are some cases with prostate cancer suspicion where TURP is able to confirm a PCa. According to our results the ratio is $12.869 \%$ [61 patients (group A + group C) from the total of 474] with newly diagnosed prostate cancer after TURP.

TURP which is still considered the gold standard of the treatment of $\mathrm{BOO}$ caused by $\mathrm{BPH}$ and by prostate cancer, is a good procedure in the diagnostic workup of $\mathrm{PCa}$, allowing a more extensive histopathological examination of the prostate, yielding a larger quantity of prostate tissue than prostate biopsy [3]. This is also explained by the multifocal nature of cancer prostate. But we also have to deal with two problems: the timing of the prostatectomy after TURP and the possibility of dissemination of the cancerous cells during TURP.

Another interesting issue is the accidental detection of PCa, in our study $6.540 \%$ (31 cases from the total of 474), in cases without suspicion of PCa preoperatively (group A). In 13 cases $(2.742 \%)$ from the total of 474 cases $\mathrm{CaP}$ was diagnosed in an early stage (1-3 small foci of prostate adenocarcinoma with Gleason scores from 4 to 6).

We consider a controversial result the cases of $11 \mathrm{pa}-$ tients (from the total of 30 patients diagnosed with $\mathrm{CaP}$ from group C) with cancer localized in the transitional zone of the prostate detected by TURP. Based on McNeal's statement, the distribution of prostate cancer shows the following: $68 \%$ starts from the peripheral zone, $8 \%$ develop from the central area, and $24 \%$ are localized in the transition zone [6]. Our point of view is that in case of patients with elevated PSA but with previous repeatedly negative prostate biopsies, we must consider the transition zone of the prostate, which is more accessible for TURP than prostate biopsy and digital rectal examination $[9,10]$.

The cases of four patients, with confirmed prostate adenocarcinoma (from group B), with negative histopathological findings after TURP, can be explained by the small focus of prostate cancer located at the peripheral zone of the prostate, inaccessible for TURP.

\section{Conclusion}

TURP remains the elective surgical therapy of the bladder outlet obstruction caused by benign prostate hyperplasia and in some cases of prostate cancer.

By yielding a greater volume of prostate tissue than prostate biopsy, TURP can aid in the detection of prostate cancer localized to the transition zone, mainly in patients with normal PSA and/or positive velocity.

In our practice, detection rate of prostate cancer with TURP performed in patients with bladder outlet obstruction $(12.869 \%)$ it is close to the literature findings (15\%)

It is a valuable option in cases with several negative ultrasound guided prostate biopsies.

\section{Conflict of interest}

None to declare.

\section{References:}

1. Dae Keun Kim, Sang Jin Kim, Hong Sang Moon, et al. The role of TURP in the detection of prostate cancer in $\mathrm{BPH}$ patients with previously negative prostate biopsy. www.kjurology.org DOI:10.4111/kju.2010.51.5.313.

2. S Radhakrishnan, TJ Dorkin, N Sheikh \& DR Greene. Role of transition zone sampling by TURP in patients with raised PSA and multiple negative transrectal ultrasound-guided prostatic biopsies. Prostate Cancer and Prostatic Diseases 2004:7;338-342.

3. Koenraad van Renterghem, Gommert Van Koeveringe, Ruth Achten, Philip van Kerrebroeck. Prospective study of the role of transurethral resection of the prostate in patients with an elevated prostate-specific antigen level, minor lower urinary tract symptoms, and proven bladder outlet obstruction. European urology 2008: 54;1385-1392.

4. Tristan Dellavedova, Rolando Ponzano, Laura Racca, Federico Minuzzi and Mariana Dominguez. Prostate cancer as incidental finding in transurethral resection. Oncologic Urology Arch. Esp. Urol. 
2010:63(10);855-861.

5. Koenraad M.L.E. van Renterghem, Philip E.V.A. van Kerrebroeck and Gommert A.Van Koeveringe. Role and current situation of turp in patients with elevated psa; Special article Arch. Esp. Urol. 2010:63(6);411-419.

6. Paolo Puppo, Carlo Introini, Paolo Calvi, Angelo Naselli. Role of transurethral resection of the prostate and biopsy of the peripheral zone in the same session after repeated negative biopsies in the diagnosis of prostate cancer. Eur Urol. 2006;49(5):873-878.

7. Djavan B. Prostate Biopsies and the Vienna Nomograms. European Urology. 2006:Suppl5;500-510.

8. Werahera PN, Sullivan K, La Rosa FG, at al: Optimization of prostate cancer diagnosis by increasing the number of core biopsies based on gland volume. International journal of clinical and experimental pathology. 2012;5:892-899.

9. Nelson AW, Harvey RC, Parker RA, et al. Repeat prostate biopsy strategies after initial negative biopsy: meta-regression comparing cancer detection of transperineal, transrectal saturation and MRI guided biopsy. PLoS One. 2013;8(2):e57480.

10. Van Renterghem K, Van Koeveringe G, Achten R, et al. A new algorithm in patients with elevated and/or rising prostate-specific antigen level, minor lower urinary tract symptoms, and negative multisite prostate biopsies. International urology and nephrology. Int Urol Nephrol. 2010;42:29-38. 